\title{
Dimensiones culturales y sustentabilidad como factores de convivencia ciudadana. Un estudio comparativo entre países
}

\author{
Cultural dimensions and sustainability as factors of citizen coexistence. A comparative \\ study between countries
}

\section{Dimensões culturais e sustentabilidade como fatores de convivência cidadã. Um estudo comparativo entre países}

Héctor Fernando Rueda Rodríguez

https://orcid.org/0000-0001-7998-0328 Universidad del Valle, sede Tuluá, Cali, Colombia

- Fecha de recepción: 2021-05-09

- Fecha concepto de evaluación: 2021-09-05

- Fecha de aprobación: 2021-09-16

https://doi.org/10.22335/rlct.v14i1.1453
Para citar este artículo / To reference this article / Para citar este artigo: Rueda-Rodríguez, $\mathrm{H}$. F. (2022). Dimensiones culturales y sustentabilidad como factores de convivencia ciudadana. Un estudio comparativo entre países. Revista Logos Ciencia \& Tecnología, 14(1), 8-17. https:// doi.org/10.22335/rlct.v14i1.1453

\begin{abstract}
RESUMEN
El objetivo del artículo es determinar la relación entre la convivencia ciudadana, el cuidado ambiental y las dimensiones culturales, a partir de un estudio documental de fuentes secundarias, entre ellas, el Environmental Performance Index (EPI) de 2020 y las dimensiones de la cultura de Hofstede de 2020. La metodología es de corte cuantitativo con alcances correlacionales; la muestra incluye países con alto desempeño ambiental, dos con desempeño ambiental medio y bajo, pero con un PIB muy alto, como es el caso de Estados Unidos y China, respectivamente, y los países de la Alianza del Pacífico. Los resultados muestran la relación entre la convivencia ciudadana, las instituciones o dimensiones culturales y el EPI. Aunque las variables estén correlacionadas, la dinámica cambiante de los aspectos institucionales implica un análisis diacrónico en contextos específicos y la necesidad de profundizaciones posteriores. No obstante, mientras la literatura sobre teoría institucional y sustentabilidad es prolífica, la correlación de variables planteada es inédita. En conclusión, el ambiente es transversal a la convivencia, y factores como el individualismo y la orientación a largo plazo la promueven, mientras la distancia de poder y la disminución de la incertidumbre la condicionan.
\end{abstract}

Palabras clave: Instituciones, cultura, sustentabilidad, coexistencia pacífica, ambiente

Correo electrónico: hector.fernando.rueda@correounivalle.edu.co 


\begin{abstract}
The objective of the article is to determine the relationship between citizen coexistence, care for the environment and cultural dimensions, based on a documentary study of secondary sources, among them, the Environmental Performance Index (EPI) of 2020 and the dimensions of the Hofstede culture of 2020. The methodology is quantitative with correlational scopes; the sample includes countries with high environmental performance, two with medium and low environmental performance, but with very high GDP, such as the United States and China, respectively, and countries of the Pacific Alliance. The results show the relationship between citizen coexistence, the institutions or cultural dimensions, and EPI. Although the variables are correlated, the changing dynamics of institutional aspects imply a diachronic analysis in specific contexts and the need of further deep research. Neverheless, while the literature on institutional and sustainability theory is prolific, the proposed correlation of variables is unedited. In conclusion, the environment is transversal to coexistence, and factors as individualism and long-term orientation promote it, while the power distance and the decrease of uncertainty, condition it.
\end{abstract}

Keywords: Institutions, culture, sustainability, peaceful coexistence, environment

\title{
RESUMO
}

O objetivo do artigo é verificar a relação entre a convivência cidadã, os cuidados ambientais e as dimensões culturais, com base num estudo documental de fontes secundárias, incluindo o Índice de Desempenho Ambiental (EPI) do 2020 e as dimensões da cultura de Hofstede do 2020. A metodologia é quantitativa com escopos correlacionais; a amostra inclui países com alto desempenho ambiental, dois com desempenho ambiental médio e baixo, mas com PIB muito alto, como é o caso dos Estados Unidos e China, respectivamente, e os países da Aliança do Pacífico. Os resultados mostram a relação entre a convivência cidadã, as instituições ou dimensões culturais e o EPI. Embora as variáveis estejam correlacionadas, a dinâmica em mudança dos aspectos institucionais implica uma análise diacrónica em contextos específicos e a necessidade de um estudo mais aprofundado . Não obstante, enquanto a literatura sobre teoria institucional e sustentabilidade seja prolífica, a correlação de variáveis proposta é inédito. Em conclusão, o ambiente é transversal à convivência, e fatores como o individualismo e a orientação a longo prazo o promovem, enquanto a distância do poder e a diminuição da incerteza o condicionam.

Palavras-chave: Instituições, cultura, sustentabilidade, convivência pacífico, ambiente

El principal aporte de esta investigación está relacionado con el entendimiento de las variables que condicionan la convivencia ciudadana o coexistencia pacífica. Para ello, se analizan las instituciones informales o las dimensiones de la cultura planteadas por Hofstede para el año 2020, en relación con indicadores como el Environmental Performance Index (EPI) de 2020, el índice de Percepción de la Corrupción (IPC) de 2019, e indicadores de la Organización para la Cooperación y el Desarrollo Económico (OCDE) de 2019. Esta relación se justifica en la medida en que los seres humanos se ajustan a su entorno natural y social, y construyen ciertos rasgos culturales que los representan, pero que condicionan su estilo de vida. En este sentido, la adaptación es el concepto clave en la relación sociedad-naturaleza. Por otra parte, en un mismo entorno o ambiente pueden aparecer distintas formas de adaptación, de ahí la diversidad cultural en un territorio. Así, puede existir una relación entre la cultura y el cuidado del ambiente, cuando ciertos rasgos ambientales específicos moldean rasgos culturales concretos (Castillo, 2015).

Numerosos autores como Evans (2004), Glaeser et al. (2004), North (2010) y León et al. (2020) evidencian cómo las instituciones conforman el marco normativo en el que se desarrollan las interacciones entre actores sociales y constituyen la estructura de incentivos dentro del campo político, económico y social. Es frecuente encontrar investigaciones alrededor del desarrollo económico y productivo, la ecología y el ambiente, y estudios de instituciones informales que no conjugan un diálogo 
interdisciplinar (Morin, 2006). La presente investigación busca revisar la interacción de conceptos de manera sistémica desde las ciencias sociales y ambientales en la resolución de problemas concretos.

Cuando hablamos de instituciones formales o informales, nos referimos a las reglas de juego que generan las personas en la sociedad (Gorodnichenco \& Roland, 2017), aquellas que moldean la interacción entre los agentes económicos, establecen parámetros y reducen los costos de transacción en las relaciones humanas. Las instituciones formales se expresan en reglamentos, leyes y normas; y las informales, se manifiestan en la cultura, hábitos y costumbres (Oyserman \& Lee, 2008). Hay formas de medir las instituciones; las formales, por ejemplo, con los indicadores de gobernanza del Banco Mundial y las informales con las dimensiones de la cultura de Hofstede (Gallén \& Peraita, 2016). Cabe anotar que las instituciones influencian directamente la actividad empresarial y la calidad de vida de las personas de un país (Baumol \& Blinder, 2008; Estrin et al., 2013; Welter \& Smallbone, 2011; Williams \& Shahid, 2016). En este sentido, se evidencia el influjo de estas sobre la sustentabilidad de la sociedad (Aragon et al., 2016).

La cultura es la relación de la sociedad con su medioambiente (Harris \& Orna, 2007). Los estudios ambientales sitúan, en un primer plano, la cultura como foco y principal aspecto regulador de la relación de las sociedades con la naturaleza (Cubillos, 2015). Desde la ecología cultural, la naturaleza y lo biofísico son objeto de conocimiento. La relación que el sujeto establezca con dicho objeto dependerá de la episteme del sujeto, de su cosmogonía o de su cultura (Leff, 2008). En la ecología cultural, las culturas y no los individuos son los que se adaptan. Steward, en las décadas de 1930 y 1940, propuso que podríamos comenzar a comprender estas adaptaciones, examinando primero el núcleo cultural, ya que este es el componente crítico que se ocupa de la capacidad de la cultura para sobrevivir (Leff, 2004).

El concepto de sociedad sustentable se ha interpretado de muchas formas, según la disciplina desde la que se aborde, su incorporación en el plano académico comenzó en la segunda mitad del siglo XX. En general, el concepto de sustentabilidad tradicional o débil está asociado a formas de producción eficientes, que disminuyen los impactos en el ambiente; en otras palabras, consumir garantizando los recursos de las generaciones futuras. Esta idea significó un gran paso en términos de justicia social, con respecto a lo que se planteaba antes de 1970 con el fenómeno de la industrialización y el crecimiento económico en auge como únicos indicadores de riqueza para los países. Entonces, se contemplaba a la naturaleza como un recurso necesario para el crecimiento económico, sin considerar la complejidad de los sistemas biológicos; se formularon políticas simples, que terminaron por agotar dicho recurso al chocar con el límite ecológico, incluyendo la pérdida de biodiversidad, la contaminación y el calentamiento global (González \& Avila, 2007; Meadows et al., 1972; Ostrom \& Cox, 2010). Por el contrario, el concepto de sustentabilidad fuerte, desde la economía ecológica y la ecología profunda, plantea a la sociedad como un actor más de la naturaleza. En este sentido, lo abiótico y lo biótico, la fauna y la flora, tienen los mismos derechos que lo antrópico, y en tal sentido, reducen las opciones de crecimiento ecológico en la medida que afecten la resiliencia de los ecosistemas (Folke, 2006; González \& Avila, 2007).

Así, el informe de Brundtland señala la necesidad de garantizar los recursos a las generaciones futuras, como es el caso de la producción de alimentos versus la preservación de los ecosistemas terrestres y acuáticos; muchas de estas acciones obedecen a políticas de Estado desarrolladas como un esfuerzo por disminuir el impacto en el ambiente y suplir las necesidades sociales, en medio del cambio climático y la crisis ambiental (López \& Ancona, 2005).

Como ya señalábamos, a nivel mundial existen indicadores que miden el desempeño en materia ambiental de los países; uno de los más importantes es el Índice de Desempeño Ambiental, EPI (por sus siglas en inglés), el cual analiza políticas ambientales y globales de 180 países, según 32 indicadores de desempeño, distribuidos en 11 categorías sobre temas que cubren desde la salud ambiental hasta la vitalidad del ecosistema. Se consideran indicadores sobre emisiones de $\mathrm{CO}_{2^{\prime}}$ generadas por el cambio en el uso del suelo y por tasas de crecimiento de las emisiones de carbono negro, saneamiento, agua potable, y contaminación del aire en interiores. El EPI utiliza como fuentes de datos a entidades como el Instituto de Métrica y Evaluación de la Salud, el Instituto de Recursos Mundiales, el Instituto Potsdam para la Investigación del Impacto Climático, CSIRO, el Grupo Mullion, y el Proyecto Sea Around Us de la Universidad de Columbia Británica, así como datos del Banco Mundial y la Organización de las Naciones Unidas para la Agricultura y la Alimentación (Environmental Performance Index, 2020). Estudiar la problemática ambiental implica entender el territorio como un lugar de convergencia interdisciplinar en 
donde se analizan factores epistemológicos, éticos, políticos, sociales o institucionales en países, ciudades o, en general, en territorios concretos, como hechos biofísicos y como producción histórica cultural, para comprender la relación entre la sociedad y la naturaleza (Fals Borda, 2000; Sáenz, 2007).

Por otra parte, las instituciones informales se pueden expresar a través de las siguientes seis dimensiones de la cultura: i) distancia del poder, 2) individualismo, 3) masculinidad, 4) disminución de la incertidumbre y 5) orientación a largo plazo (Hofstede, 2001). Estudios sobre cultura (Hall et al., 2013) analizan, entre otras, la relación con la autoridad; el concepto de uno mismo, concretamente la relación entre el individuo y la sociedad; el concepto individual de masculinidad y de feminidad; $y$ las maneras de tratar con los conflictos, incluyendo el control de la agresión y de la expresión de los sentimientos.

La convivencia ciudadana implica desarrollar estrategias a partir de un mapeo confiable de la realidad local (Echevarría et al., 2012). Al igual que en los sistemas biológicos, un entorno sustentable implica menores presiones para la supervivencia de las especies o individuos en la sociedad; investigaciones sugieren que, a pesar de la disposición de los sujetos hacia la reconciliación, la tolerancia y el respeto a nivel retórico, cuando se trata de la interacción en contextos específicos, factores ideológicos, estéticos y ambientales, estos los condicionan (Michalinos, et al, 2011). En coherencia con los sistemas biológicos, la resiliencia de los ecosistemas humanos depende de la diversidad de los recursos, la capacidad de los individuos de asumir las funciones sociales y el nivel de adaptación a la perturbación ocasionada por eventos adversos; para el caso de Colombia, las perturbaciones provienen principalmente de conflictos sociales. En este sentido, el país ha visto los resultados de más de 50 años de conflicto armado, que tienen diferentes manifestaciones dependiendo del contexto en donde se ubiquen, bien sea rural o urbano. Para el caso de las principales ciudades, se han consolidado grupos organizados de delincuencia, que se disputan territorialmente el control de las rentas criminales; esta situación afecta el diario vivir de los habitantes de la ciudad, deteriorando el tejido social, pues generan una cultura del irrespeto por la autoridad, incluida la constitución política y las normas básicas de convivencia (Torrente, 1998).

Ante un panorama social tan caótico, es menester explorar otros contextos sociales, representados por otras culturas, e indagar cómo el desempeño ambiental, económico e institucional genera una estructura de incentivos que promueve la coexistencia pacífica de la ciudadanía.

Al revisar la literatura disponible se constata que, hasta el momento, no son evidentes estudios con igual correlación de variables; aunque los artículos de León et al. y Wendling et al. (2020) resaltan la relación entre las instituciones y el desempeño social y ambiental.

\section{Metodología}

La investigación es un estudio documental de fuentes secundarias con alcances correlacionales. La muestra de países se definió a juicio del investigador e incluye 12 países, divididos en tres grupos, que representan formas culturales y políticas sociales, económicas y ambientales diferentes. Entre ellos se encuentran países con un alto desempeño ambiental, dos países con desempeño ambiental medio y bajo, pero con un PIB muy alto, como es el caso de Estados Unidos y China, respectivamente, y los países de la Alianza del Pacífico. Estos últimos fueron elegidos teniendo en cuenta que tienen identidades culturales compartidas, debido a la historia y a los antepasados coloniales. A continuación, se correlaciona el Índice de Desempeño Ambiental (Environmental Performance Index, 2020), además de otros indicadores como el índice de Percepción de la Corrupción (IPC, 2019), Panorama de la educación, indicadores de la Organización para la Cooperación y el Desarrollo Económico (OCDE, 2019); con seis dimensiones culturales de Geert Hofstede (2019) por cada país:

a. Países de la Alianza del Pacífico (México, Perú, Colombia y Chile), excolonias españolas cuyo EPI va desde el nivel medio al bajo.

b. Seis países que tienen el EPI más alto (Dinamarca, Luxemburgo, Suiza, Reino Unido, Francia y Australia), de los cuales cinco se encuentran ubicados en el norte de Europa y uno es una excolonia británica.

c. Estados Unidos y China, dos potencias económicas e industriales que representan las políticas del capitalismo occidental y el socialismo oriental; el índice EPI de estos países está en un término medio y muy bajo, respectivamente.

A continuación, se describen las dimensiones culturales planteadas por Hofstede (2001), que se utilizaron para medir el nivel institucional de 0 a 100, por cada uno de los 12 países: 
- Distancia de poder: cuanto más alto el puntaje, mayor distancia de poder. En estas sociedades es aceptable que el estado, representado por un grupo de personas privilegiadas, ostente el poder y ejerzan autoridad formal, en ellas, los mecanismos de veeduría ciudadana a la función pública son insuficientes, lo que facilita actos de corrupción (Hosftede, 2011). Así, los países con baja dimensión de poder muestran mejores niveles de desarrollo sostenible, que aquellos que tienen alta dimensión de poder, pues estos "consideran que las normas y leyes deben ser consensuadas e incluir los intereses de la mayoría" (Hofstede, 2011, p. 9).

- Individualismo/colectivismo: cuanto más alto el puntaje, mayor individualismo. Estas sociedades propenden por el desarrollo en las comunidades de autonomía política y administrativa que favorezcan los intereses del colectivo en detrimento de los intereses particulares (Fals Borda, 2000)

- Masculinidad/feminidad: cuanto más alto el puntaje, mayor injerencia masculina en la sociedad. Esto tiene que ver principalmente con el papel que juegan los individuos en la sociedad, dependiendo de su género. En cuanto a los valores, la masculinidad representa la competitividad, mientras la feminidad representa la cooperación (Hofstede, 2011). Por otra parte, es beneficioso incluir a la mujer en el plano político, ya que representa mayor grado de inclusión, lo cual es positivo para la sociedad (Unión Interparlamentaria y ONU, 2014).

- Disminución de la incertidumbre: cuanto mayor sea el puntaje en esta dimensión, mayor reducción de la incertidumbre. Estas sociedades estarán menos dispuestas a tomar riesgos.

- Orientación a largo/corto plazo: Entre mayor es la orientación al largo plazo predomina la cultura del ahorro y la preparación para el futuro, son en general sociedad más pragmáticas.

- Indulgencia: define la medida en que las personas intentan controlar sus deseos e impulsos, en función de la forma en que fueron educados: entre mayor sea el puntaje, más indulgente es un país. Este tipo de sociedades muestran una disposición a realizar sus impulsos y deseos con respecto al placer y a la diversión, lo que les impulsa a actuar como les place, aunque esto signifique pasar por encima de las libertades de los otros, lo cual fomenta una mala convivencia y el irrespeto por las autoridades.

Al final, se correlaciona el EPI versus seis dimensiones institucionales para cada uno de los 12 países. En este análisis, un índice cerca a uno (1) indica una correlación positiva de la variable institucional estudiada. Como ejemplo, en la primera dimensión, una mayor distancia de poder se relacionaría con un EPI más bajo. Si el índice se acerca a menos uno (-1) indica una correlación negativa de la variable institucional estudiada. En este sentido, en la primera dimensión, una menor distancia de poder se relacionaría con un EPI más alto. Por otra parte, un índice lejano de uno (1) o de menos uno (-1) indica que la dimensión institucional no es lo suficientemente significativa para variar el EPI.

\section{Resultados y discusión}

El análisis implica la correlación de las seis variables propuestas por Hofstede con indicadores de desempeño a nivel social y ambiental:

\section{Distancia de poder}

Existe relación entre el respeto a la autoridad formal y los índices de corrupción; así lo demuestra un estudio que toma como muestra a más de 150 países, en el que, además, se resalta la necesidad de robustecer las instituciones (Castañeda, 2016).

La figura 1, muestra que países con mayor distancia de poder tienen un menor EPI. Es el caso de los países de la Alianza del Pacífico y China, que tienen una distancia de poder alta y el EPI, en promedio, más bajo. Además, si se revisa el índice de Percepción de la Corrupción (IPC, 2019), se identifica una relación muy similar entre la corrupción institucional, la censura, la vigilancia continua y la represión a los ciudadanos y el EPI (Sorman, 2006).

Figura 1

Comparación de la distancia de poder y EPI por países 2020

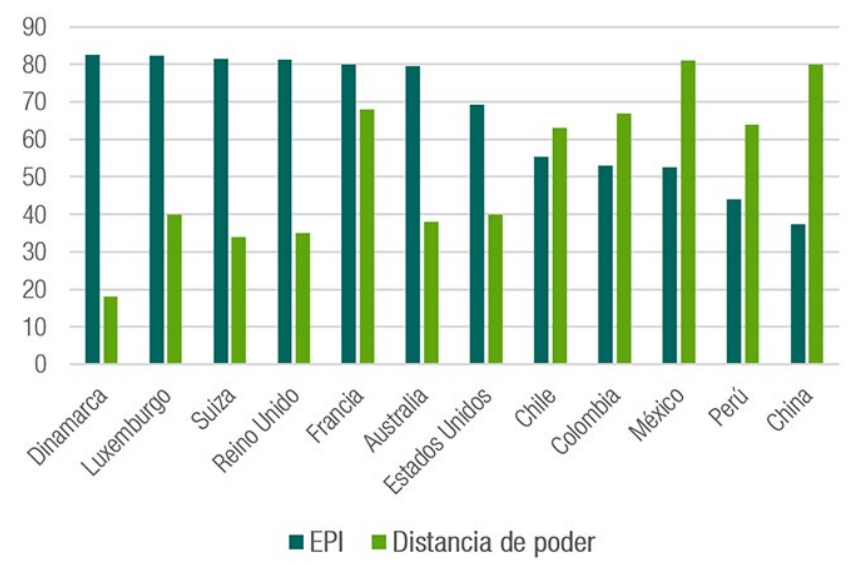

Nota. La figura muestra cómo países con mayor distancia de poder tienen un menor EPI. Es el caso de los países de la Alianza del Pacífico y China. Tomado de Hofstede Insights (2020) y Environmental Performance Index (2020). 


\section{Individualismo/Colectivismo}

La figura 2 muestra que países con mayor individualismo tienen un mayor EPI. Los países de la Alianza del Pacífico y China tienden a ser más colectivistas y en promedio tienen un EPI más bajo.

Una manifestación del colectivismo es el bajo nivel de educación formal de un país. Al revisar el panorama de la educación para estos países, expuesto por los indicadores de la Organización para la Cooperación y el Desarrollo Económico (OCDE, 2019), se encuentra una relación directa entre bajo nivel de formación profesional, falta de educación política, desarrollo investigativo y el EPI.

Figura 2

Comparación del individualismo y el EPI por países 2020

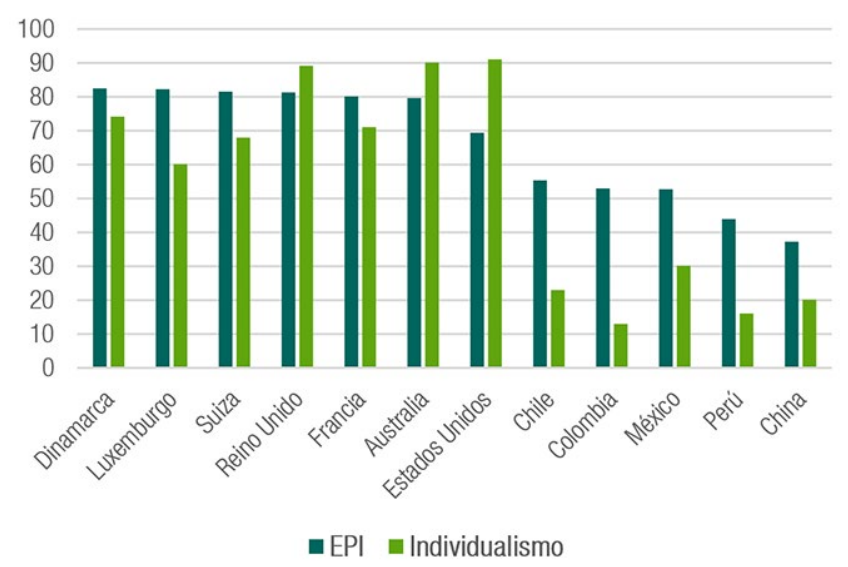

Nota. La figura muestra cómo países con menor individualismo tienen menor EPI. Es el caso de los países de la Alianza del Pacífico y China. Tomado de Hofstede Insights (2020) y Environmental Performance Index (2020).

\section{Masculinidad}

La figura 3 muestra que los países con menor masculinidad tienen en promedio un mayor EPI. Es el caso de Dinamarca, en Europa, y Chile, por la Alianza del Pacífico; seguidos por Perú, Francia, Luxemburgo, Australia, Estados Unidos, Colombia, Reino Unido, China, México y Suiza. Los resultados muestran una heterogeneidad por regiones, aunque también es resaltable que dos países con EPI muy bajos como México y China tengan los índices de masculinidad más altos.

\section{Disminución de la incertidumbre}

La figura 4 muestra que países con mayor reducción de la incertidumbre tienen un menor EPI. Para el caso de los países de la Alianza del Pacífico, China y Francia tienden a evitar la incertidumbre $y$, en promedio, tienen un EPI más bajo, a excepción de Francia que cuenta con un EPI alto.
Figura 3

Comparación de la masculinidad y el EPI por países 2020

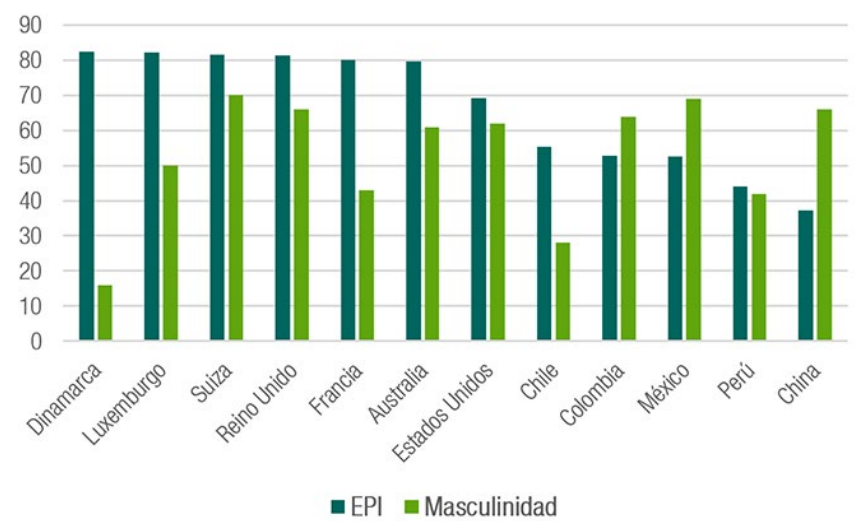

Nota. La figura muestra países con menor masculinidad tienen en promedio un mayor EPI. Es el caso de Dinamarca, por Europa y Chile. Tomado de Hofstede Insights (2020) y Environmental Performance Index (2020).

La utilización de combustibles fósiles como sustento de las demandas energéticas es una apuesta segura desde lo económico, teniendo en cuenta las reservas de petróleo de grandes potencias económicas, a diferencia de países desarrollados sustentablemente o en vía de desarrollo como Colombia, que asume el riesgo de utilizar energías alternativas, lo cual es un factor clave en la minimización de gases con efecto invernadero (Chavarro et al., 2017). Esto muestra que sociedades que aceptan los cambios y la incertidumbre como algo natural en su desarrollo, tienden a tener una mejor calidad de vida que aquellas que se muestran reacias y temerosas a afrontar cambios (Heskitalo et al., 2016).

Figura 4

Comparación de la disminución de la incertidumbre y el EPI por países 2020

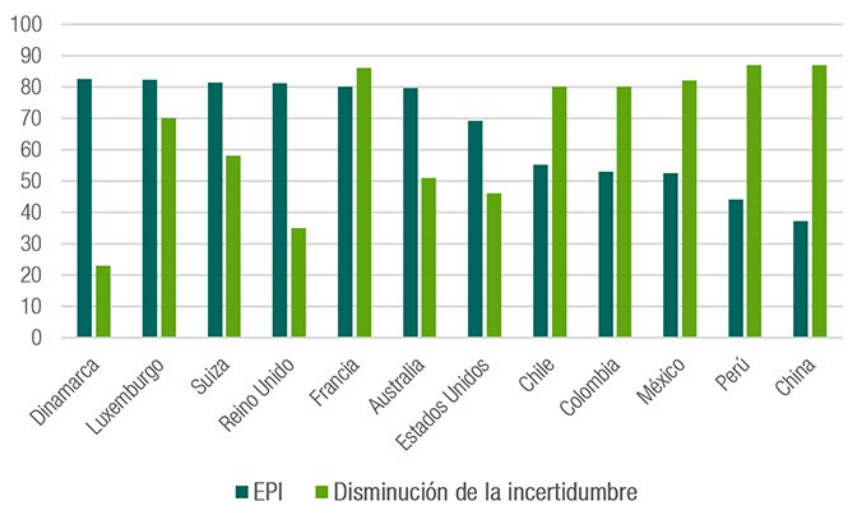

Nota. La figura muestra países con mayor reducción de la incertidumbre tienen un menor EPI. Para el caso de los países de la Alianza del Pacífico, China y Francia tienden a evitar la incertidumbre. Tomado de Hofstede Insights (2020) y Environmental Performance Index (2020) 


\section{Orientación a largo/corto plazo}

La figura 5 muestra la relación entre el desarrollo sostenible y la orientación a largo plazo. A excepción de Australia, los países sustentables orientan sus políticas en el largo plazo; esto tiene mucho sentido, en la medida en que cuidar el medio ambiente es, en general, una apuesta a futuro, algo muy similar a ahorrar recursos ambientales (Waissbluth, 2021). En los países de la Alianza del Pacífico y China se observa una orientación marcada hacia el corto plazo; los eventos más importantes en la vida ocurrieron en el pasado o toman lugar ahora, existen certezas sobre lo que es bueno y lo que es malo, las tradiciones son sacrosantas, se debe estar orgulloso del país de origen y el Estado opera en favor del crecimiento económico por encima del desarrollo sostenible, ya que muestra resultados en cuanto a bienestar de forma inmediata (Hofstede, 2011).

Para el caso de los países desarrollados, se observa una marcada tendencia al largo plazo, con excepción de Estados Unidos que muestra un indicador similar a los países de la Alianza del Pacífico y China. Lo anterior demuestra que las tradiciones son adaptables, así como los modelos económicos; se trata de aprender de otros países, el ahorro y la perseverancia son importantes (Waissbluth, 2021).

\section{Figura 5}

Comparación de la orientación al largo plazo y el EPI por países 2020

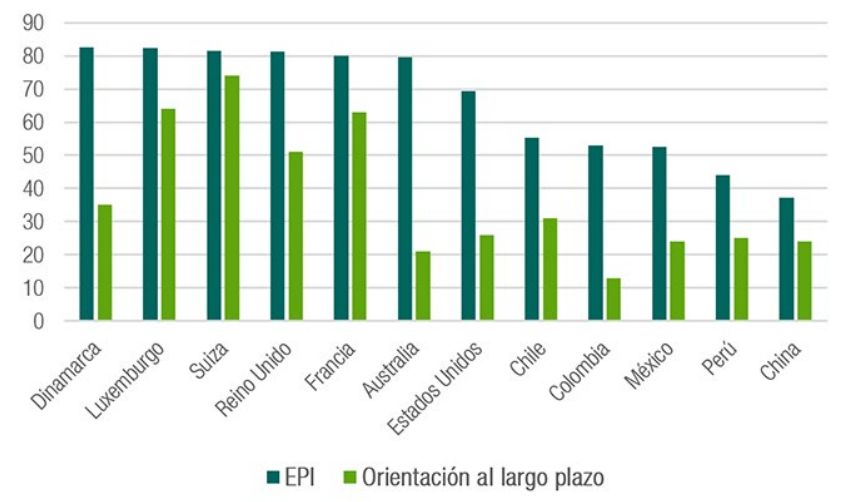

Nota. El gráfico muestra que los países con mayor orientación a largo plazo tienen un mayor EPI, a excepción de Australia. En promedio, los países sustentables orientan sus políticas a largo plazo. Tomado de Hofstede Insights (2020) y Environmental Performance Index (2020).

\section{Indulgencia/restricción}

Las cifras de indulgencia son muy altas para México (97) las cifras se encuentran en un promedio de (63), lo cual es un indicador de sana convivencia, como era de esperarse. China obtiene la calificación más baja (24) lo cual es una posible manifestación de las restricciones a nivel

normativo y de la expresión cultural de su población, después de años de vivir en un régimen autoritario en el que predominan las estrategias de control a la población (Kalathil, 2017).

Figura 6

Comparación de la indulgencia y el EPI por países 2020

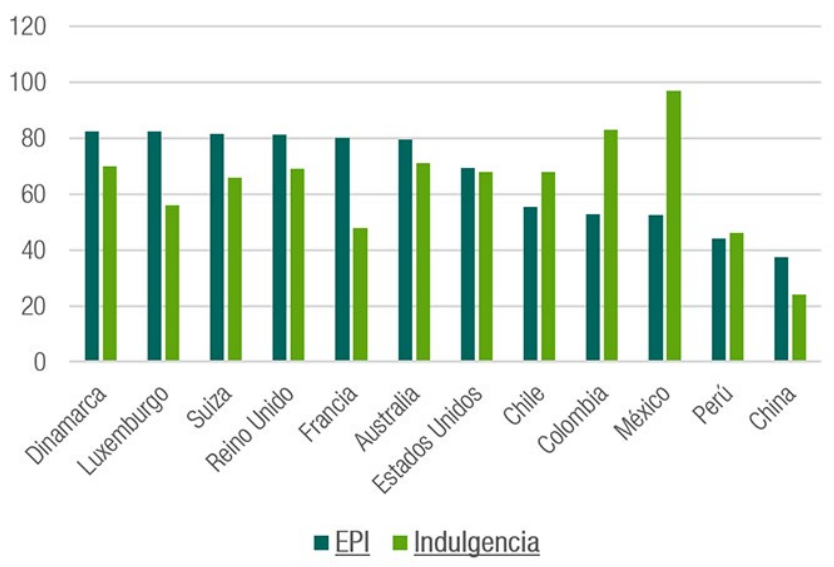

Nota. El gráfico muestra que países con mayor indulgencia tienen un menor EPI. Las cifras de indulgencia son muy altas para México (97) y Colombia (83). Tomado de Hofstede Insights (2020) y Environmental Performance Index (2020).

\section{Correlación de variables}

Aunque las diferencias entre países no son estáticas sino dinámicamente consistentes, debido a la prominencia del individualismo y el colectivismo (Oyserman \& Lee, 2008), la correlación permite observar algunas tendencias, entre ellas, que, a mayor distancia de poder, menor $\mathrm{EPI}$; a mayor individualismo, mayor EPI; para el caso de la masculinidad versus la feminidad, los resultados son muy heterogéneos por regiones y las diferencias no son significativas. En cuanto a la disminución de la incertidumbre, a mayor valor, menor EPI; a mayor orientación al largo plazo, mayor EPI y a menor indulgencia, mayor EPI.

Se resalta el alto desempeño EPI en correlación con instituciones informales, tales como: distancia de poder, individualismo, incertidumbre, orientación a largo plazo e indulgencia. Esta última muestra de países como México y Colombia, además de compartir elementos culturales y un desempeño ambiental similar, se relaciona con la norma de forma conflictiva.

Los procesos sostenibles ambientalmente y el desarrollo de métodos de apropiación territorial de naturaleza económica, acordes con la identidad cultural (Fals Borda, 1999; Lozano, 2009; Torres Carrillo, 2009), se generan a partir de iniciativas colectivas de base, algunas de ellas articuladas a procesos de intervención comu- 
nitaria, cuyo propósito es fortalecer las capacidades asociativas y organizativas a través de procesos educativos y de investigación. En este caso, la población se involucra activamente en el estudio de sus problemas, a partir de la generación de diagnósticos sociales, la priorización de problemas colectivos y la planificación participativa de acciones, de tal forma que, los oriente, conjuntamente con equipos técnicos, a visibilizar y plantear nuevas condiciones de sana convivencia y coexistencia pacífica.

Tabla 1

Correlación de variables

\begin{tabular}{|c|c|c|c|c|c|c|c|}
\hline Países & EPI & $\begin{array}{l}\text { Distancia } \\
\text { de poder }\end{array}$ & $\begin{array}{l}\text { Individualismo } \\
\text { vs. colectivismo }\end{array}$ & $\begin{array}{l}\text { Masculinidad } \\
\text { vs. feminidad }\end{array}$ & $\begin{array}{c}\text { Disminución } \\
\text { de la incertidumbre }\end{array}$ & $\begin{array}{l}\text { Orientación a largo } \\
\text { / corto plazo }\end{array}$ & Indulgencia \\
\hline Dinamarca & 82.5 & 18 & 74 & 16 & 23 & 35 & 70 \\
\hline Luxemburgo & 82.3 & 40 & 60 & 50 & 70 & 64 & 56 \\
\hline Suiza & 81.5 & 34 & 68 & 70 & 58 & 74 & 66 \\
\hline Reino Unido & 81.3 & 35 & 89 & 66 & 35 & 51 & 69 \\
\hline Francia & 80 & 68 & 71 & 43 & 86 & 63 & 48 \\
\hline Australia & 79.6 & 38 & 90 & 61 & 51 & 21 & 71 \\
\hline EUA & 69.3 & 40 & 91 & 62 & 46 & 26 & 68 \\
\hline Chile & 55.3 & 63 & 23 & 28 & 86 & 31 & 68 \\
\hline Colombia & 52.9 & 67 & 13 & 64 & 80 & 13 & 83 \\
\hline México & 52.6 & 81 & 30 & 69 & 82 & 24 & 97 \\
\hline Perú & 44 & 64 & 16 & 42 & 87 & 25 & 46 \\
\hline China & 37.3 & 80 & 20 & 66 & 87 & 24 & 24 \\
\hline Correlación & & -0.802614049 & 0.866714756 & -0.12871845 & -0.686911443 & 0.663491708 & 0.22105917 \\
\hline
\end{tabular}

Nota. La tabla muestra como la correlación de variables permite observar algunas tendencias. Tomado de Hofstede Insights (2020) y Environmental Performance Index (2020)

\section{Conclusiones}

Existen indicios de una relación directamente proporcional entre algunas de las dimensiones institucionales planteadas por Hofstede y el EPI; entre ellas se encuentran el individualismo y la orientación a largo plazo, dado que, al revisar el indicador de desarrollo sostenible, las diferencias son evidentes entre países con dimensiones institucionales con una calificación alta.

Para otras dimensiones institucionales como la distancia de poder y la reducción de la incertidumbre, la relación es inversamente proporcional con el EPI. Aunque las variables estén correlacionadas, existe un espacio para profundizaciones posteriores, sobre todo teniendo en cuenta la dinámica cambiante de los aspectos institucionales; además, en medio de los cambios ambientales que vive el planeta, es imperativo explorar factores institucionales que favorezcan el cuidado de los recursos naturales y la preservación de los ecosistemas.
La calidad de vida de la población pasa por proveer un ambiente saludable en el que no solo prime el crecimiento económico sino también el cuidado del medioambiente y el bienestar de la población. En este sentido, las instituciones juegan un papel importante en la regulación social necesaria para su sostenimiento.

La sana convivencia es un indicador que mide la tolerancia y el respeto por los espacios del otro. Los países con un EPI muy alto mantienen un equilibrio entre el libre actuar y las restricciones, lo cual también se expresa en índices de desarrollo humano y calidad de vida elevados.

La población debe participar activamente en el diagnóstico, priorización de problemas colectivos y la planificación participativa de acciones, de tal forma que los ayude a visibilizar y a plantear condiciones de sana convivencia. 


\section{Referencias}

Aragon, J. Pardo, M., \& Roig, S. (2016). La influencia de desarrollo de las instituciones en la decisión de creación de empresa: una visión cognitiva. Revista de Investigación Empresarial, (69), 4941-4946. https://doi. org/10.1016/j.jbusres.2016.04.056

Baumol, W. J., \& Blinder, A. (2008). Macroeconomía: principios y política. Cincinnati, $\mathrm{OH}$ : suroeste.

Castañeda, V. (2016). Una investigación sobre la corrupción pública y sus determinantes. Revista Mexicana de Ciencias Políticas y Sociales, (61), 103-135. https:// doi.org/10.1016/S0185-1918(16)30023-X

Castillo, L. (2015). Conflictos ambientales y movimientos sociales: el caso del movimiento embera katío en respuesta a la construcción de la represa Urrá (19942008). Memoria y Sociedad, 19(39), 94-105.

Chavarro, D., Vélez, M., Tovar, G., Montenegro, I., Hernández, A., \& Olaya, A. (2017). Los objetivos del desarrollo sotenible en Colombia y el aporte de la ciencia, la tecnología y la innovación. https://minciencias.gov. co/sites/default/files/ctei_y_ods_-_documento_de_trabajo.pdf

Cubillos, L. (2015). Los estudios socioculturales como estrategia académica para la comprensión de las problemáticas ambientales del territorio. Ambiente y Sostenibilidad, (5), 36-42.

Echevarría, L., Lopera, C., Morales, N., Urán, S., \& Arango, C. (2012). Sistema de información para la seguridad y la convivencia: una buena práctica en análisis delictual para la ciudad de Medellín, Colombia. En F. P. Ciudadana, Buenas prácticas para el análisis delictual en América Latina 2012 (pp. 75-86). Medellín.

Environmental Performance Index. (2020). https://epi. yale.edu/

Estrin, S., Korosteleva, J., \& Mickiewicz, T. (2013). Which institutions encourage entrepreneurial growth aspirations? Journal of Business Venturing, 28(4), 564-580.

Evans, P. (2004). Development as institutional change: The pitfalls of monocropping and the potentials of deliberation. Studies in Comparative International Development, 38, 30-52.

Fals Borda, O. (1999). Orígenes universales y retos actuales de la IAP. Análisis Político, (38), 73-90.

Fals Borda, O. (2000). El territorio como construcción social. Revista Foro, 38, 45-58.
Foladori, G., \& Naína, P. (2005). ¿Sustentabilidad? desacuerdos sobre el desarrollo sustentable. Colección América Latina y el nuevo orden mundial. Miguel Ángel Porrúa, UAZ, Cámara de Diputados LIX Legislatura.

Gallén, M., \& Peraita, C. (2016). Información de responsabilidad social corporativa y teoría institucional. Intangible Capital, 12(4), 942-977.

Glaeser, E., La Porta, R., \& Lopez-de-Silanes, F. (2004). Do Institutions Cause Growth? Journal of Economic Growth, 9, 271-303.

González, C., \& Avila, VS., (2019). Operacionalización y medición de la resiliencia socioecológica: una revisión sistemática. Sostenibilidad, 11(21): 60-73. https:// doi.org/10.3390/su11216073

Gorodnichenco, Y., \& Roland, G. (2017). Cultura, instituciones y la riqueza de las naciones. Review of Economics and Statistics, 99(3), 402-416.

Hall, S., Walsh, C., Restrepo, E., \& Vich, V. (2013). Sin garantías: trayectorias y problemáticas en estudios culturales. Corporación Editora Nacional, CEN; Universidad Andina Simón Bolívar, Sede Ecuador; Pontificia Universidad Javeriana. Instituto de Estudios Sociales y Culturales Pensar; Instituto de Estudios Peruanos.

Harris, M., \& Orna J. (2007). Antropología Cultural, (7 ed.). Pearson.

Hofstede Insights. (2019). Compare countries. https://www. hofstede-insights.com/product/compare-countries/

Hofstede, G. (1997). Culture and Organizations Software of the Mind. Mc Graw Hill. https://seryactuar.files. wordpress.com/2015/08/3-culturas-y-organizacioneshofstede.pdf

Hofstede, G. (2001). Culture's consequences: Comparing values, behaviors, institutions and organizations across nations. Sage Publications.

Hofstede, G. (2011). Dimensionalizing Cultures: The Hofstede Model in Context. Psychology and Culture, 2(1), 1 -26. https://doi.org/10.9707/2307-0919.1014

Kalathil, S. (2017). Beyond the Great Firewall: How China Became a Global Information Power, Cima, National Endowment for Democracy. https://www.cima.ned. org/wp-content/uploads/2017/03/CIMA-Beyond-theGreat-Firewall_150ppi-for-web.pdf

Keskitalo, E., Bergh, J., Felton, A., Björkman, C., Berlín, M., Axelsson, P., Anillo, E., Ågren, A., Roberge, J., Klapwijk, M., Boberg, J. (2016) Adaptación al cambio cli- 
mático en la silvicultura sueca. Forest, 7: 2-28. https:// doi.org/10.3390/f7020028

Leff, E. (2004). Racionalidad ambiental. La apropiación social de la naturaleza. Siglo XXI Editores.

Leff, E. (2008). Discursos Sustentables. Siglo XXI Editores.

León, C., Rueda, H., \& González, C. (2020). Instituciones formales, desarrollo humano y emprendimiento: un estudio comparativo entre países con alto nivel de desarrollo y los países de la Alianza del Pacífico. $A D$ minister, (36), 45-66.

López, E., \& Ancona, I. (2005). Desarrollo sustentable o sostenible: una definición. Horizonte Sanitario, 4(2), $1-7$.

Lozano, F. H. (ed). 2009. Herramientas de manejo para la conservación de biodiversidad en paisajes rurales. Instituto de Investigación de Recursos Biológicos Alexander von Humboldt y Corporación Autónoma Regional de Cundinamarca (CAR).

Meadows, D. H., Meadows, J., Randers, W.W., \& Beherens, I. (1972). Los límites del crecimiento. Fondo Cultura Económica.

Morin, E. (2006). El Método VI: Ética. Ediciones Cátedra.

Michalinos, Z., Costandina, Ch., Panayiota, Ch., \& Panayiota, K. (2011). Promoting peaceful coexistence in conflict-ridden Cyprus: Teachers' difficulties and emotions towards a new policy initiative. Teaching and Teacher Education, 27(2), 332-341.

North, D. (2010). Instituciones cambio institucional y desempeño económico. Fondo de Cultura Económica.

Organización para la Cooperación y el Desarrollo Económico [OCDE] (2019). Education at a Glance 2019, Organisation for Economic Co-Operation and Development (OCDE). https://www.observatoriodelainfancia.es/oia/esp/documentos_ficha.aspx?id=5861

Ostrom, E., \& Cox, M. (2010). Moving beyond panaceas: a multitiered diagnostic approach for social ecological analysis. Environmental Conservation, 37(4), 451-463.

Ostrom, E. (2011). El gobierno de los bienes comunes. La evolución de las instituciones de acción colectiva. UNAM / IIS /CRIM / FCE.

Oyserman, D., \& Lee, S. (2008). Diversidad étnica y preferencias por la redistribución. Boletín Psicológico, 134(2), 311-342.
Sáenz, E. (2007). La ofensiva empresarial: industriales, políticos y violencia en los años 40 en Colombia. Universidad Nacional de Colombia.

Sorman, G. (2006). China: el imperio de las mentiras. (1 ${ }^{\text {a }}$ ed.). Ed. Sudamericana,.

Torrente, D. (1998). La sociedad policial: poder, trabajo y cultura en una organización local de policía. ( $1^{\mathrm{a}}$ ed.). Centro de investigaciones Sociológicas.

Torres Carrillo, A. (2009). Vigencia y perspectivas de la investigación participativa. MEDIACIONES, 7(9), 173183. https://doi.org/10.26620/uniminuto.mediaciones.7.9.2009.173-183

Transparency Internacional. (sf.) IPC 2019: Resumen global. https://www.transparency.org/es/news/cpi2019-global-highlights

Unión Interparlamentaria y ONU (2014). Hechos y cifras: Liderazgo y participación política de las mujeres. ONU Mujeres. http://www.unwomen.org/es/what-wedo/leadership-and-political-participation/facts-andfigures\#sthash.fb9zi20n.dpuf

Welter, F., \& Smallbone, D. (2011). Perspectivas institucionales sobre el comportamiento empresarial en entornos desafiantes. Journal of Small Business Management, 49(1), 107-125.

Waissbluth, M. (2021). Introducción a la gestión pública. Publicado por Penguin Random House Grupo Editorial. https://books.google.es/books?id=yv8gEAAAQB AJ\&lpg=PT6\&ots=W04Bo-vD5K\&lr\&hl=es\&pg=PP1 \# $\mathrm{v}=$ onepage $\& \mathrm{q} \& \mathrm{f}=\mathrm{false}$

Wendling, Z.A. Warren, D.C., Rubin, B.M., Carley, S., \& Richards, K.R. (2020). A Scalable Energy-Economy Model for State-Level Policy Analysis Applied to a Demand-Side Management Program. Economic Development Quarterly, 34(4), 372-386. https://doi. org/10.1177/0891242420937792

Williams, C., \& Shahid, M. (2016). Emprendimiento informal y teoría institucional: explicación de los distintos grados de (in) formalización de los empresarios en Pakistán. Emprendimiento y Desarrollo Regional, 28(1-2), 1-25. 